\title{
New device and technique to protect intubation operators against COVID-19
}

\author{
Chaojin Chen ${ }^{1,2}$, Ning Shen ${ }^{1 *} \mathbb{D}$, Xiaoyun $\mathrm{Li}^{1}$, Qi Zhang ${ }^{2}$ and Ziqing Hei ${ }^{1 *}$
}

(0) 2020 Springer-Verlag GmbH Germany, part of Springer Nature

Dear Editor,

Over 200 countries have now reported more than 1.7 million laboratory-confirmed cases of novel coronavirus disease 2019 (COVID-19) [1], and thousands of healthcare workers have been infected as a result of shortages of personal protective equipment (PPE) [2]. Most severe or critical COVID-19 cases eventually require endotracheal intubation to improve ventilation [3]. Given that intubation operators need to get close to the patient's nose and mouth, and respiratory droplets containing the virus might land on their faces and clothes [4], it is urgently necessary to use practical devices to protect them from direct contact and also potential self-contamination when doffing PPE; such practical devices are especially needed in countries which lack PPE [5].

To address this challenge for intubation operators, we describe a convenient new protective device designed to make tracheal intubation safer. The device is essentially a clear plastic cover serving to isolate the patient's exhaled gas and droplets (Fig. 1a). Using this protective sleeve, the neck and head of COVID-19 confirmed or suspected patients could be covered to create a relatively closed environment. All the operations would be carried out through three holes: one for the mask, one for the laryngoscope blade, and one for the intratracheal tube (Fig. 1b, c). This convenient device not only enables us to avoid direct contact with the patients' droplets, but also reduces the risk of self-contamination when doffing PPE.

To better demonstrate the isolation effect of the protective sleeve, we first attempted endotracheal intubation in a human tracheal-intubation model (HTM). Green

*Correspondence: paulshenyh@163.com; heiziqing@sina.com

${ }^{1}$ Department of Anesthesiology, The Third Affiliated Hospital, Sun Yat-Sen University, No.600 Tianhe Road, Guangzhou, People's Republic of China

Full author information is available at the end of the article phosphor was poured into the lung of the HTM to highlight exhaled airflow. As shown in Fig. 1d and Video S1, when we squeezed the lung of the HTM to simulate human coughing during intubation, the sleeve could effectively isolate the HTM's expired droplets. After that, we used the protective sleeve in patients. Water-sensitive paper (WSP) was pasted on its inner and outer sides to test for the presence of droplets (Figure S1A). We found that it had a satisfactory effect in isolating the patients' droplets without causing hypoxemia during intubation, as shown by the fact that only the inner WSP received droplets during the procedure (Figure S1 and Table S1). The preparation of the protective sleeve and the endotracheal intubation procedure are shown in Videos S2 and S3.

The protective sleeve is modified from an aseptic microscopy cover, and it is simple to prepare and use. In fact, any similar sleeve with good airtightness can be used to obtain the same function. This protective sleeve can also be used to improve the safety of healthcare workers who perform sputum aspiration, bronchoscopy and broncho-alveolar lavage in confirmed or suspected COVID-19 patients in the ICU, emergency department and operating room. In addition, we suggest that the protective device should be applied in all patients undergoing general anesthesia and tracheal intubation during the COVID-19 epidemic because of the potential for symptomatic infections among patients. We think that this protective sleeve may play a surprising role in protecting first-line doctors against COVID-19 infection, especially in developing countries and territories where there is an extreme dearth of PPE against COVID-19.

\section{实


A

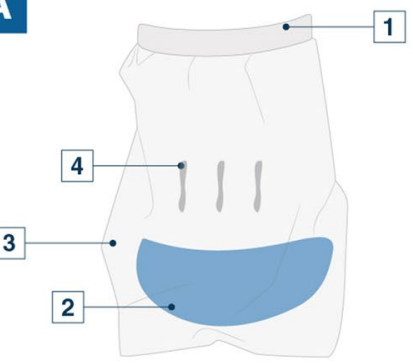

B

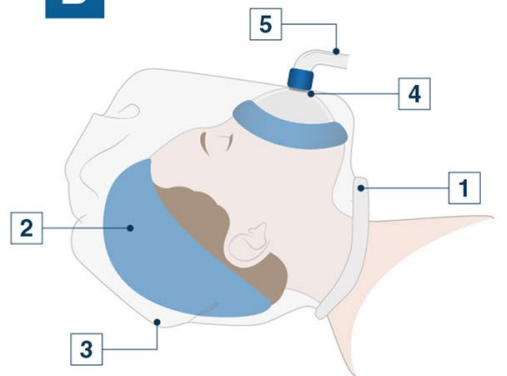

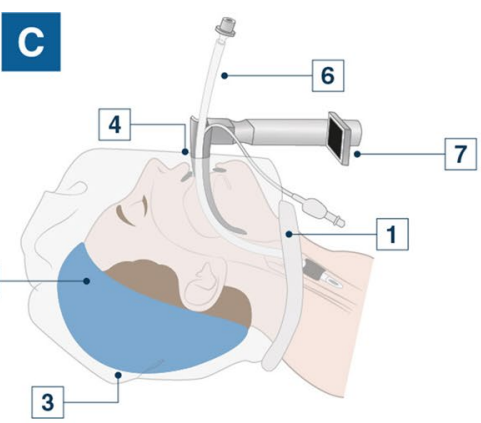

D
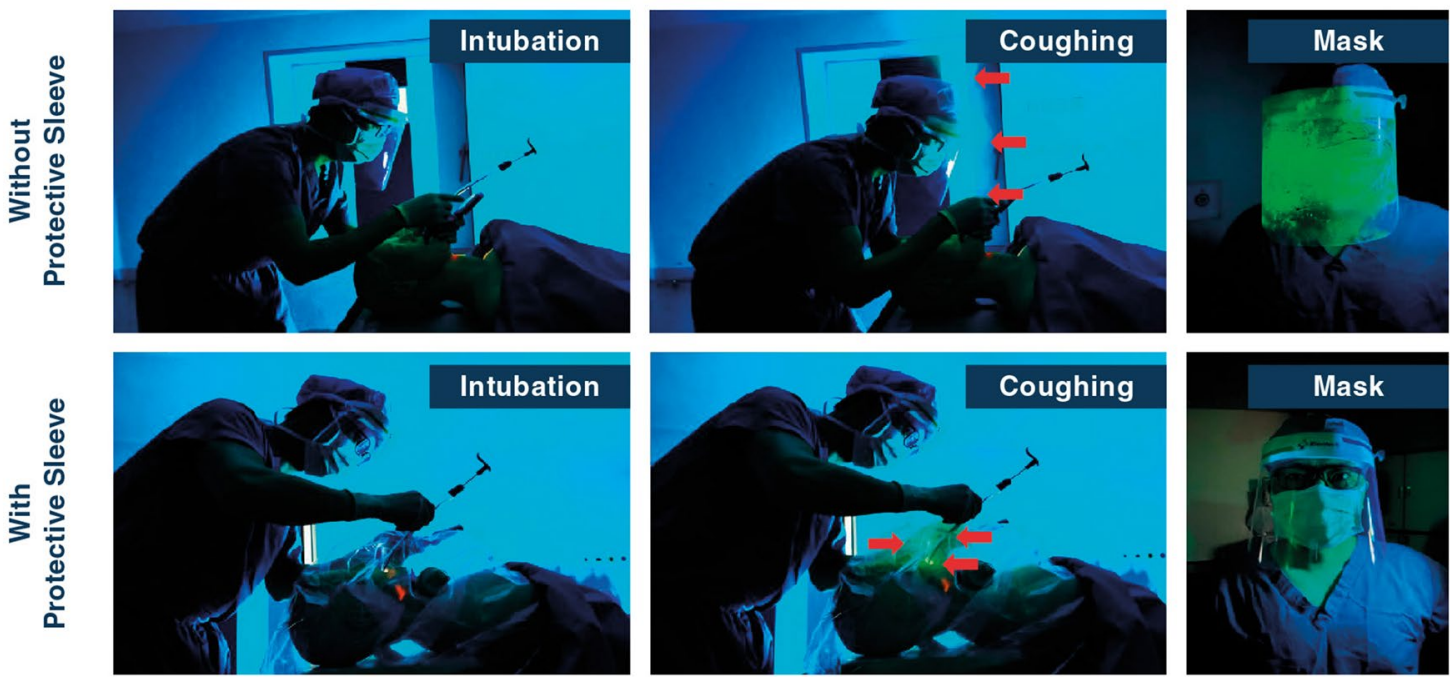

Fig. 1 Isolation of respiratory droplets with the protective sleeve during intubation. a Schematic diagram of the self-made endotracheal intubation protective sleeve; $\mathbf{b}$ mask ventilation with the protective sleeve; $\mathbf{c}$ intubation with the protective sleeve; 1 . elastic belt; 2 . head covering; 3 . neck covering; 4. operating hole; 5. mask; 6. endotracheal tube; 7. video laryngoscope; d Endotracheal intubation was attempted in an HTM (left). We then squeezed the lung of the HTM to simulate human coughing (middle). The respiratory droplets from the HTM were highlighted by the green phosphor (right). HTM, human tracheal-intubation model; Red arrow: green phosphor

\section{Electronic supplementary material}

The online version of this article (https://doi.org/10.1007/s00134-020-06072-9) contains supplementary material, which is available to authorized users.

\section{Author details}

${ }^{1}$ Department of Anesthesiology, The Third Affiliated Hospital, Sun Yat-Sen University, No.600 Tianhe Road, Guangzhou, People's Republic of China.

${ }^{2}$ Cell-gene Therapy Translational Medicine Research Center, The Third Affiliated Hospital, Sun Yat-Sen University, No.600 Tianhe Road, Guangzhou, People's Republic of China.

\section{Acknowledgements}

This study is supported by the National Natural Science Foundation of China (Grant No. 81772127), Postdoctoral Science Foundation of China (Grant No. 2019M663260) and the Medjaden Academy \& Research Foundation for Young Scientists (Grant No. COVID-19-MJA20200301).

\section{Authors' contribution}

CC and NS designed the protective sleeve. CC, NS and ZH contributed to the experimental design. CC, NS, XL and QZ carried out the experiment. CC and QZ searched literature and wrote the paper. CC and ZH revised the manuscript and all the authors approved the final version.

\section{Compliance with ethical standards}

\section{Conflicts of interest}

We declare no competing interests.

\section{Publisher's Note}

Springer Nature remains neutral with regard to jurisdictional claims in published maps and institutional affiliations.

\section{Accepted: 24 April 2020}

Published online: 8 May 2020

\section{References}

1. WHO (2020) Coronavirus disease 2019 (COVID-19) Situation Report-84 13 April 2020. https://www.who.int/docs/default-source/coronaviruse/ situation-reports/20200413-sitrep-84-covid-19.pdf?sfvrsn=44f511ab_2

2. WHO (2020) Shortage of personal protective equipment endangering health workers worldwide. March 3, 2020. https://www.who.int/ news-room/detail/03-03-2020-shortage-of-personal-protective-equip ment-endangering-health-workers-worldwide 
3. Alhazzani W, Møller M, Arabi Y et al (2020) Surviving Sepsis Campaign: guidelines on the management of critically ill adults with coronavirus disease 2019 (COVID-19). Intensive Care Med. https://doi.org/10.1007/ s00134-020-06022-5

4. Chen C, Hei Z, Xing J et al (2019) Laryngoscopic techniques modulate anaesthesiologists' perception of halitosis in patients: a randomised controlled trial. Eur J Anaesthesiol 36:918-923
5. Michael B, Daniel J, Eli N (2014) Ebola virus disease and the need for new personal protective equipment. JAMA 312:2495 